\title{
High performance matrix inversion of SPD matrices on graphics processors
}

\author{
Peter Benner*, Pablo Ezzatti ${ }^{\dagger}$, Enrique S. Quintana-Orti ${ }^{\ddagger}$ and Alfredo Remón ${ }^{\ddagger}$ \\ ${ }^{*}$ Max-Planck-Institute for Dynamics of Complex Technical Systems \\ Magdeburg, Germany \\ Email: benner@mpi-magdeburg.mpg.de \\ ${ }^{\dagger}$ Centro de Cálculo-Instituto de Computación \\ Universidad de la República \\ Montevideo, Uruguay \\ Email: pezzatti@fing.edu.uy \\ ${ }^{\ddagger}$ Dept. de Ingeniería y Ciencia de los Computadores \\ Universidad Jaime I \\ Castellón, Spain \\ Emails\{quintana,remon\}@icc.uji.es
}

\begin{abstract}
We introduce high performance implementations of the inversion of a symmetric positive definite matrix. Two alternatives are studied and evaluated, the traditional approach based on the Cholesky factorization and the Gauss-Jordan elimination algorithm. Several implementations of the two algorithms are developed on a hybrid architecture equipped with a general-purpose multi-core processor and a graphics processor. Numerical experiments show the efficiency attained by the proposed implementations on the target architecture.
\end{abstract}

Keywords-matrix inversion; symmetric positive definite matrices; graphics processors; multi-core processors; high performance.

\section{INTRODUCTION}

Though the explicit inversion of matrices can often be by-passed by solving systems of linear equations, there are situations where the inverse of a matrix itself is of interest. Examples include earth sciences [1], the matrix sign function method for spectral decomposition [2], and other disciplines (see [3] for a detailed list). Here, we focus on the inversion of symmetric positive definite (SPD) matrices. This operation, like many other dense linear algebra computations, requires an important computational effort in terms of execution time and memory, which motivates the usage of graphics processors (GPUs) [4], [5], [6].

In this paper we analyze two different methods for computing the inverse of an SPD matrix, the traditional approach based on the Cholesky factorization, and the Gauss-Jordan elimination algorithm, more suitable for massively parallel architectures such as GPUs. Several high performance implementations of both methods and three different architectures (a multi-core CPU, a graphics processor, and an hybrid CPU-GPU architecture) are presented and evaluated. Numerical experiments illustrate the efficiency attained by the Gauss-Jordan elimination implementations on the target hybrid platform, composed of a multi-core CPU and a single GPU.
The rest of the paper is structured as follows. In Section II we review the two approaches for computing the inverse of an SPD matrix. Several high performance implementations of each method are described and evaluated in Sections III and IV. Finally, a few concluding remarks and future work are offered in Section V.

\section{HIGH PERFORMANCE MATRIX INVERSION OF SYMMETRIC POSITIVE DEFINITE MATRICES}

Two different methods for the inversion of SPD matrices are evaluated in this section, the traditional approach that uses the Cholesky factorization, and the approach based on the Gauss-Jordan elimination method [7].

Essentially, both algorithms perform the same operations; but they exhibit different properties, with the GJE method being more appealing from the point of view of high performance and parallelization.

\section{A. Matrix inversion based on the Cholesky factorization}

The traditional approach to compute the inverse of an SPD matrix $A \in \mathbb{R}^{n \times n}$ is based on the Cholesky factorization and consists of the three following steps:

1) Compute the Cholesky factorization $A=U^{T} U$, where $U \in \mathbb{R}^{n \times n}$ is an upper triangular matrix.

2) Invert the triangular factor $U \rightarrow U^{-1}$.

3) Obtain the inverse from the matrix-matrix product $U^{-1} U^{-T}=A^{-1}$.

Exploiting the symmetry of $A$, the computational and storage cost of the algorithm are significantly reduced. In particular, the computational cost is $n^{3}$ floating-point arithmetic operations, flops (compared, e.g., with the $2 n^{3}$ flops required to invert a nonsymmetric matrix). In-place inversion of the matrix (i.e., inversion using only the storage provided in $A$ ) is possible which, only references the upper triangular part of the matrix. However, for performance, $A$ is stored as a full $n \times n$ matrix. 
B. Matrix inversion based on the Gauss-Jordan elimination algorithm

The Gauss-Jordan elimination (GJE) algorithm is, in essence, a reordering of the computations performed by the traditional approach. Thus, it has the same computational cost.

Although the GJE requires the same computational cost, it sweeps through the matrix only once, reducing the number of memory accesses [8]. This is a very important property in order to obtain a high performance implementation in current architectures, where a single memory operation takes considerably more time than several floating-point operations. Moreover, most of the computations are cast in terms of matrix-matrix products, which is a highly parallel operation. Therefore, the GJE algorithm is a suitable method for the new massively parallel architectures (e.g., the GPUs)

Alike the method based on the Cholesky factorization, the GJE can also exploit the symmetric structure of the matrix to reduce the number of flops. A careful implementation can also compute the inverse without using more memory than the one provided in the initial matrix.

Two blocked variants of this algorithm are shown in Tables I and II. In both variants, the active block, $A_{11}$, moves along the main diagonal. Only the upper part of the matrix is computed, and the elements bellow the main diagonal are not referenced. The algorithms are specified using the FLAME notation [9] [10]. There, $m(\cdot)$ stands for the number of rows of its argument; TRIU $(\cdot)$ returns the upper triangular part of a matrix; " $\star$ " specifies blocks in the lower triangular part of the matrix, which are not referenced. We believe the rest of the notation is intuitive. Next to each operation, we provide the name of the BLAS kernel that is employed to perform the corresponding computation.

In the first variant (see Fig. I), at a given iteration, the elements placed on the right of block $A_{11}$ are not updated/referenced. Eight operations are carried out, six of them matrix-matrix products. However there are some features that will have a negative effect on the performance:

- Data dependencies serialize the execution of most of the operations (i.e., four operations update the same block, $\left.A_{11}\right)$. Thus, concurrency is limited to the inner parallelism of each operation.

- All the operations, except the update of $A_{00}$, involve only small blocks (taking into account that, for performance reasons, the block size $b$ is chosen to be small compared with $n$ ). In fact, even $A_{00}$ is a small block during the first iterations of the loop.

A solution could be to set the block-size $b$ to a large value; this would increment the size of the operations and also their inner parallelism. But, usually, $b$ is chosen to be small for performance reasons, as that allows an efficient use of the memory hierarchy present in current architectures, and thus, a higher performance.

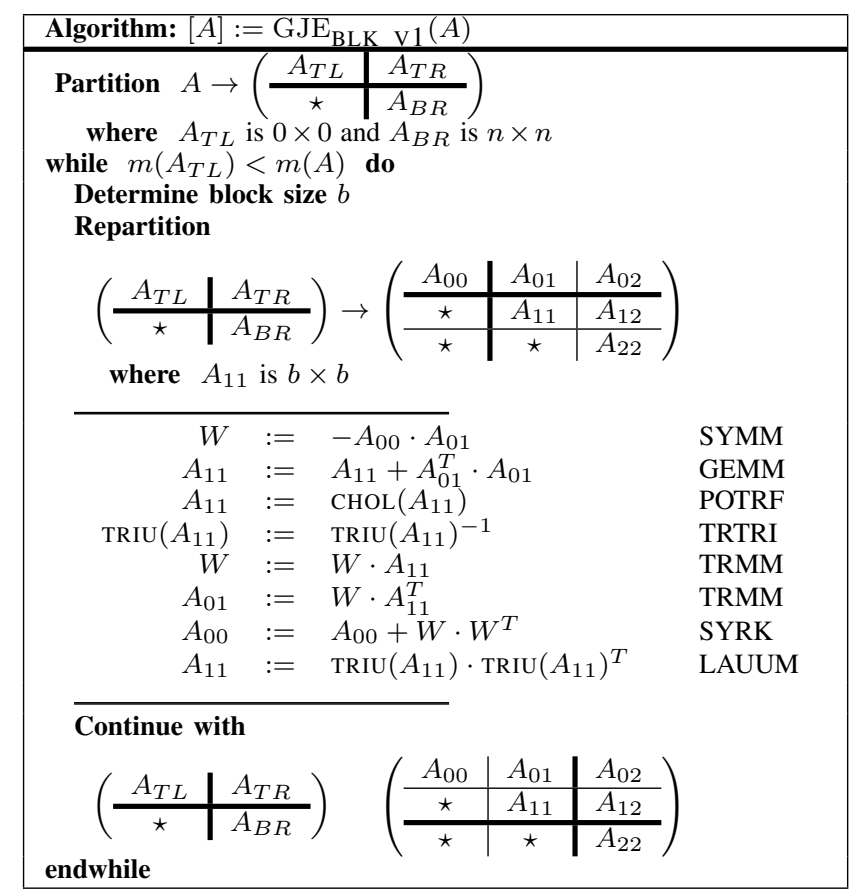

Table I

BLOCKED ALGORITHM FOR MATRIX INVERSION OF SPD MATRICES VIA GJE (VARIANT 1).

Table II shows a second variant of the GJE algorithm more suitable for parallel computing. In this variant, at each iteration, all the elements in the upper part of the matrix are updated. Although data dependencies among the operations still exist, in this case, two operations involve large blocks and concentrate most of the computations (taking into account that, for performance reasons, $b$ is "small"), namely, the updates of blocks $A_{00}$ and $A_{22}$. In particular, both computations are performed as matrix-matrix products, which is well-known to be a highly parallel operation.

One more remarkable aspect of the second variant is that it does not need any additional workspace, while the first variant utilizes an $n \times b$ workspace.

\section{HIGH PERFORMANCE IMPLEMENTATIONS}

\section{A. Implementations based on the Cholesky factorization}

The algorithm based on the Cholesky factorization for the computation of the inverse of an SPD matrix (see Section II-A) is composed of three steps that must be executed sequentially. This implies that parallelism has to be extracted inside the execution of each step.

Three high performance implementations for this algorithm are described next. All of them compute the inverse in-place, reducing the memory requirements.

1) Implementation on a multi-core CPU: the Intel MKL library [11] offers kernels for the Cholesky factorization of an SPD matrix (routine potrf, Step 1), and the inversion 


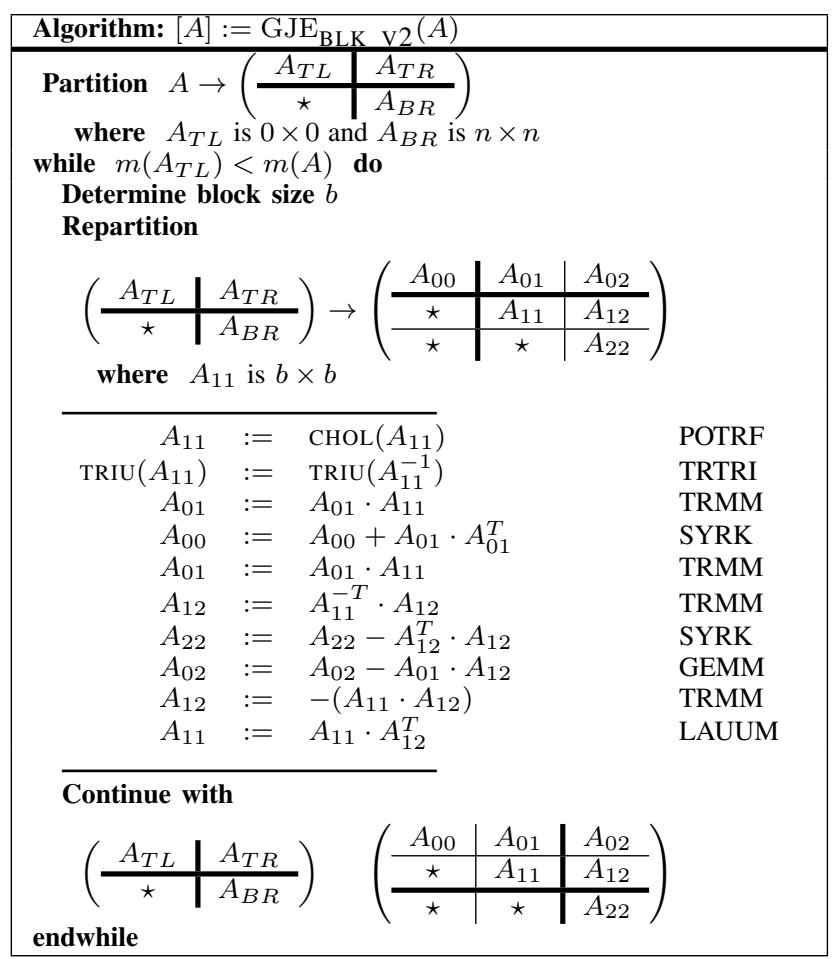

Table II

BLOCKED ALGORITHM FOR MATRIX INVERSION OF SPD MATRICES VIA GJE (VARIANT 2).

using its triangular factors (routine potri, Steps 2 and 3). The use of a multi-thread version of MKL offers parallelism and efficiency on the execution of both routines in a multicore CPU.

2) Implementation on a many-core GPU: this implementation is similar to the previous one but the target architecture is now a GPU. Currently, there are no LAPACK [12] implementations available for GPUs, so one of our main contributions in this work is the implementation of routines, gpu_potrf and gpu_potri from LAPACK for the GPU architecture. The first one computes the Cholesky factorization (Step 1), while gpu_potri can be used to obtain the matrix inverse from its Cholesky factors. The implementation of both routines makes intensive use of kernels from the nVidia CUBLAS library [13].

This implementation requires some data transfers between the CPU and the GPU memory spaces. Initially the matrix to invert is transferred to the GPU; the inverse is then computed in the GPU; and finally the inverse is retrieved back to the CPU.

3) Hybrid Implementation: this implementation executes each operation on the most convenient device with a minor increase of data transfers between them. In general, the CPU is faster than the GPU executing fine grain operations, while the GPU outperforms the CPU in the execution of large and highly parallel operations. Our algorithm is mainly composed of large operations suitable for the GPU, but it also performs a few fine grain operations, specially during the computation of the Cholesky factorization. In this implementation, the CPU and the GPU work jointly to compute the Cholesky factorization of the matrix; in particular, the large matrix-matrix products (symmetric rank$k$ updates) that appear during the Cholesky factorization, are computed by the GPU, and the rest of the operations are executed on the CPU.

\section{B. Implementations based on the Gauss-Jordan elimination}

In this subsection we describe five implementations for the GJE algorithm shown in Section II.

1) Implementation on a multi-core CPU: two variants of the GJE algorithm were presented in Section II-B. In both variants, most of the computations are cast in terms of matrix-matrix products. In particular, the operation that involves a higher number of flops is a symmetric rank- $k$ update. The MKL library offers highly tuned implementations of this kernel as well as of the remaining operations present in algorithms $\mathrm{GJE}_{B L K_{-} V 1}$ and $\mathrm{GJE}_{B L K_{-} V 2}$. Routines CPU_GJE_v1 and CPU_GJE_v2 implement those algorithms using MKL kernels. Parallelism is obtained, once more, within the execution of each single operation invoking the multi-threaded version of MKL.

2) Implementation on a many-core GPU: as most of the operations of the GJE algorithm can be cast in terms of matrix-matrix products, high performance can be expected from an implementation of the algorithms in Tables I and II tuned for GPUs.

In this version, routines GPU_potrf and GPU_potri are used in the update of block $A_{11} \in \mathbb{R}^{n b \times n b}$, which is a relatively small block. Therefore, we cannot expect any significant benefit from computing them in the GPU. Nevertheless, the purpose of this is to reduce the amount of data transfers between the GPU and the CPU memory spaces.

3) Hybrid implementation: high performance can be expected from the GPU implementations as the algorithms mostly invoke kernels which are available in the CUBLAS library. However, some fine grain computations, like the update of block $A_{11}$, can limit performance since they are not suitable for this architecture. The hybrid routine described in Table III implements the $\mathrm{GJE}_{B L K_{-} V 2}$ algorithm which carries out the update of block $A_{11}$ on the CPU and the rest of the computations on the GPU.

In this implementation, each operation is executed on the most convenient device, with a small overhead due to data transfers. This ensures optimal performance in each single operation while the data communication time remains small. Furthermore, both architectures perform computations concurrently when possible, reducing the computational time. 


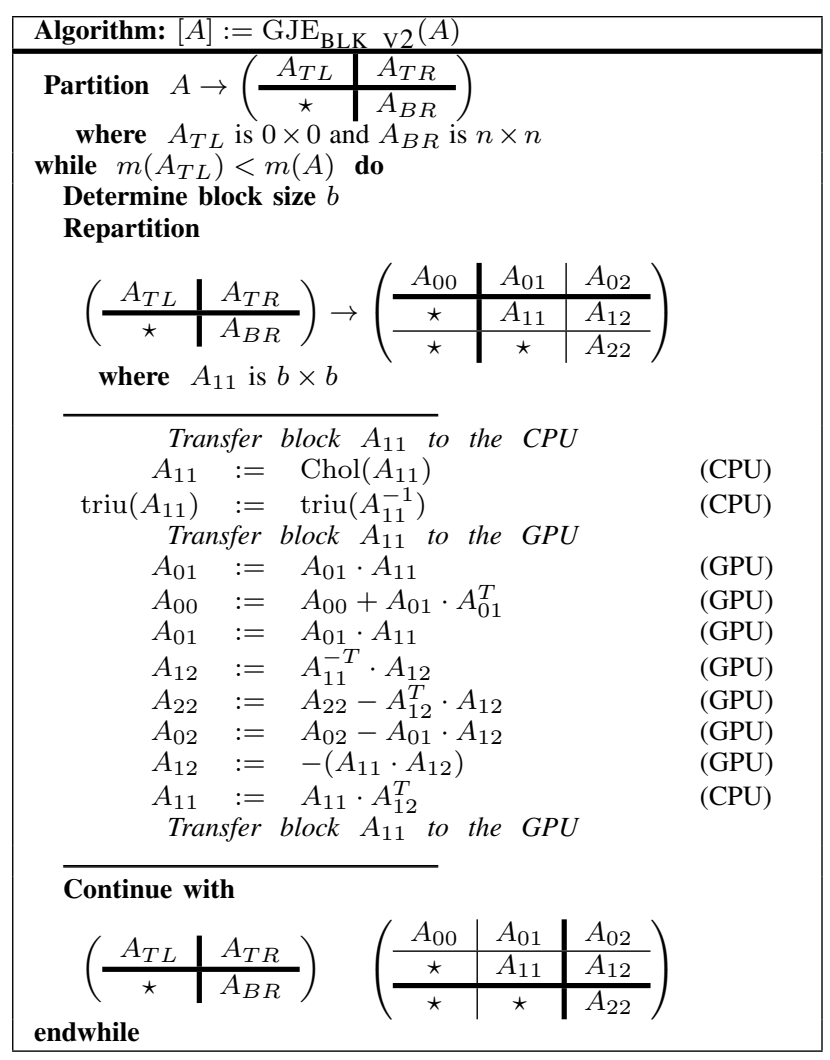

Table III

HYBRID CONCURRENT IMPLEMENTATION FOR MATRIX INVERSION OF SYMMETRIC POSITIVE MATRICES VIA GJE (VARIANT 2) IN A HYBRID ARCHITECTURE COMPOSED BY A MULTICORE CPU AND A GPU.

Three transfers are needed per iteration, all involving a small block, $A_{11}$. Those transfers permit to execute each operation on the most convenient architecture, and the concurrent execution of operations on the CPU and on the GPU, adding a new level of parallelism and improving the resources utilization.

\section{EXPERIMENTAL RESULTS}

In this section we evaluate the implementations presented in Section III and compare their performance with that of the routine in LAPACK.

All experiments were performed using IEEE singleprecision arithmetic. Results are shown for symmetric positive definite matrices of dimension $1,000,2,000, \ldots, 15,000$. Different algorithmic block sizes were tested $(512,256,128$, 64 and 32) but, for simplicity, only the performance obtained with the optimal algorithmic block size is shown.

The platform employed in the experiments consists of four Intel Xeon X7550 processors (8 cores per processor) connected to an nVidia S2050 GPU via a PCI-Express 2.0 bus. Although the nVidia device is composed of 4 GPUs (nVidia C2050, Fermi), with up to 448 cores per GPU, only

\begin{tabular}{|l||c|c|c|c|}
\hline Processors & \#cores & $\begin{array}{c}\text { Freq. } \\
(\mathrm{GHz})\end{array}$ & $\begin{array}{c}\text { L2 } \\
(\mathrm{MB})\end{array}$ & $\begin{array}{c}\text { Memory } \\
(\mathrm{GB})\end{array}$ \\
\hline \hline Intel Xeon X7550 & $\begin{array}{c}(8 \times 4) 32 \\
448\end{array}$ & $\begin{array}{c}2.0 \\
1.15\end{array}$ & $\begin{array}{c}18 \\
-\end{array}$ & $\begin{array}{c}129 \\
6\end{array}$ \\
nVidia C2050 & 48 \\
\hline
\end{tabular}

Table IV

HARDWARE EMPLOYED IN THE EXPERIMENTS.

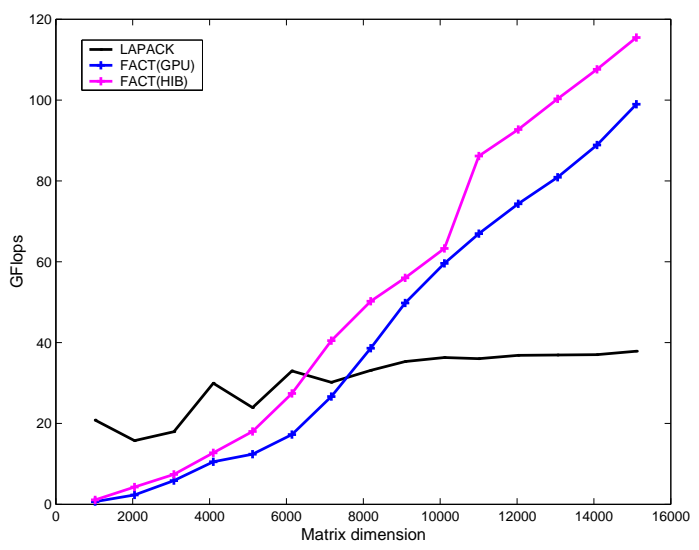

Figure 1. Performance of the matrix inversion codes based on the Cholesky factorization.

one of the GPUs is employed in the experiments. Further details on the hardware can be found in Table IV

Kernels from the Intel MKL 11.0 multi-threaded implementation of BLAS are used for most of the computations on the multi-core, while routines from nVidia CUBLAS 3.2 are employed on the GPU.

Figure 1 shows the performance attained by the implementations based on the Cholesky factorization. The LAPACK implementation obtains the best performance for matrices of dimension up to 6,000 while, for larger matrices, implementations for the graphic processor clearly outperform LAPACK. The hybrid approach increases the performance notoriously, becoming $3 \times$ faster than the traditional LAPACK implementation for matrices of dimension 15,000. Furthermore, while LAPACK seems to reach its highest performance on matrices of dimension 10,000, the performance of the GPU-based implementations continues growing even for the larger matrices evaluated in the experiment $(15,000)$. Thus, the use of a massively parallel architecture, like the GPU, increases the scalability of the implementation.

Figure 2 shows the performance achieved by the different implementations based on the GJE algorithm. The best performance is again obtained with the hybrid variant. The multi-core implementations obtain also remarkable performances, specially the routine that implements the second variant of the algorithm. In contrast, the implementations that only use the graphic processor deliver a poor performance. This is due to the number of operations in the algorithm that involve small blocks. Those operations are not 


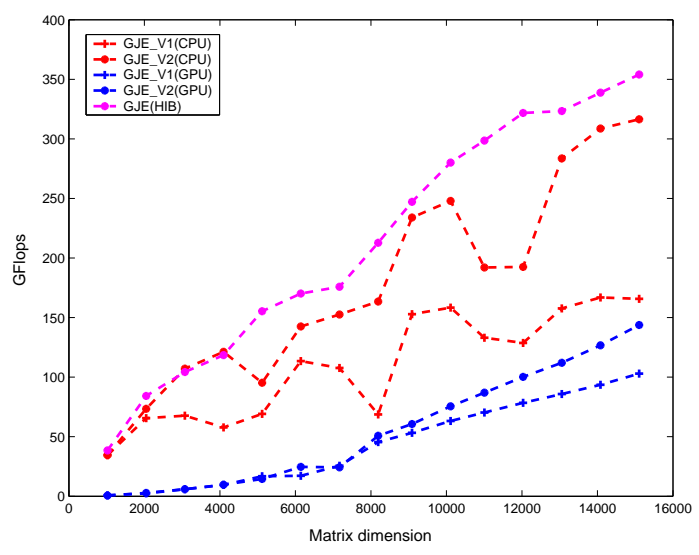

Figure 2. Performance of the matrix inversion codes based on the GJE algorithm.

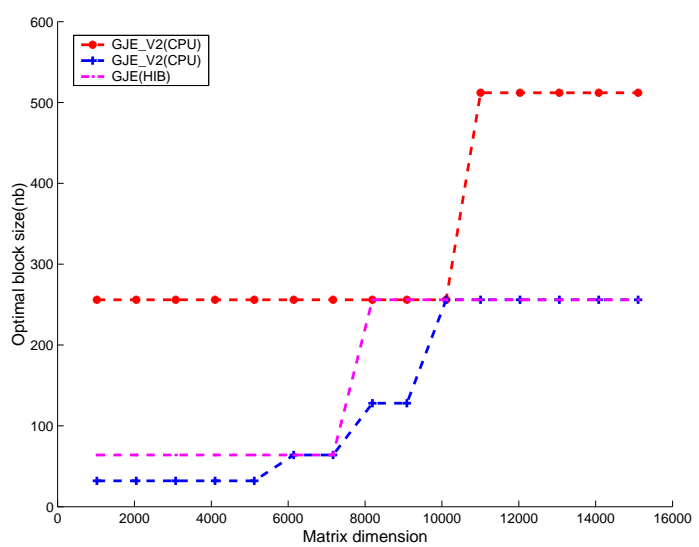

Figure 3. Optimal algorithmic block size.

well suited for the GPU architecture, so high performance cannot be expected from their execution. The consequence is that the execution time dedicated to perform small operations is too high and, consequently, the performance of the overall process suffers. On the other hand, the multi-core efficiently executes the operations that involve small blocks, so, despite this architecture is less efficient for the large operations, the overall performance is higher. We can observe this in Figure 3. The GJE_v2(CPU) implementation attains its highest performance with a large block size (256 or 512), while GJE_v2(GPU) achieves its best results with a small block size (32 to 256). When the block size is reduced, the execution time of the small operations also decreases. The hybrid approach is somewhere in the middle between the other two alternatives: the optimal block size moves from 64 to 256, since it aims to balance the execution time of the small operations on the multi-core and the large operations on the GPU.

Finally, Figure 4 shows the performance of the LAPACK implementation and the best implementations for each archi-

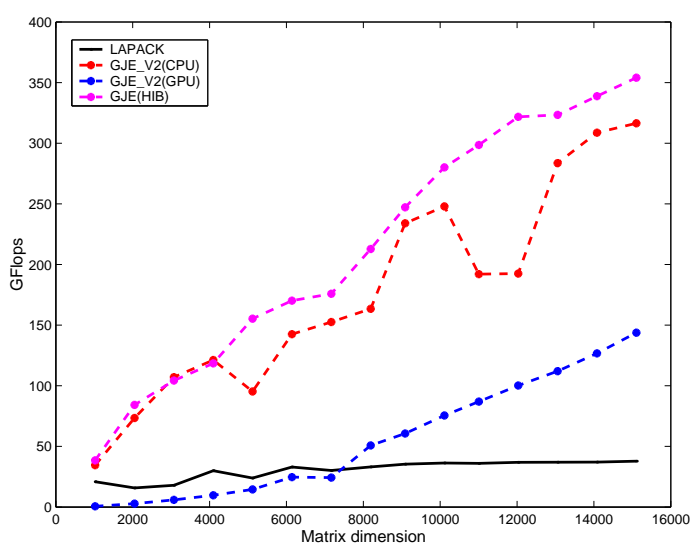

Figure 4. Performance of the matrix inversion codes.

tecture. It is important to notice that, for all the architectures, the best implementation is based on the GJE algorithm. Routine GJE_v2(GPU) outperfoms LAPACK when the matrix dimension is larger than 7,000. With smaller matrices, the data transfer time and the operations with small blocks yields it inefficient. The multi-core implementation, GJE_v2(CPU), offers an irregular behaviour, but clearly outperforms LAPACK. The fastest option is the hybrid implementation based on the GJE algorithm; despite the communication overhead, it executes each operation on the most convenient device, and also performs concurrent operations on both devices, so it offers higher performance than any other multi-core implementation. We would like to remark the scalability of the GJE(HIB) variant, and, in general, of all the implementations based on the GJE algorithm.

\section{CONCLUDING REMARKS}

We have analyzed the inversion of SPD matrices. This operation appears in different scientific applications and features a high computational cost, which asks for the use of high performance architectures like multi-core CPUs or graphics processors (GPUs). The study includes the evaluation of two different algorithms, the conventional one based on the Cholesky factorization, and the GJE algorithm, more suitable for parallel architectures.

Several implementations are presented for each algorithm and architecture. Most of the computations are executed invoking kernels from high performance libraries, Intel's MKL library in the case of the multi-core CPU, and the nVidia CUBLAS library for the GPU.

Experimental results show that higher performance is attained from those routines based on the GJE algorithm. This algorithm exhibits a remarkable scalability in all its implementations. Three different hardware options are addressed: multi-core CPU, GPU, and a hybrid platform consisting of a multi-core CPU connected to a GPU. The best performance is obtained with the hybrid implementations, where both 
architectures work jointly to compute the matrix inverse. The main advantage of the hybrid implementations comes from the execution of each operation on the most convenient device, and the concurrent usage of both resources.

\section{ACKNOWLEDGMENTS}

The authors would like to thank Francisco Igual for his technical support, and Manuel Ujaldon from the University of Malaga (Spain) for granting the access to the hardware facilities employed in the performance evaluation.

Enrique S. Quintana-Ortí and Alfredo Remón were supported by the CICYT project TIN2008-06570-C04.

This work was partially performed while Alfredo Remón and Pablo Ezzatti were visiting the Max Plank Institute (MPI) in Magdeburg. Pablo Ezzatti gratefully acknowledges support received from the MPI.

\section{REFERENCES}

[1] B. Tapley, B. Schutz, and G. Born, Statistical Orbit Determination. Elsevier Academic Press, 2004.

[2] J. Roberts, "Linear model reduction and solution of the algebraic Riccati equation by use of the sign function," vol. 32, pp. 677-687, 1980, (Reprint of Technical Report No. TR-13, CUED/B-Control, Cambridge University, Engineering Department, 1971).

[3] N. J. Higham, Accuracy and Stability of Numerical Algorithms, 2nd ed. Philadelphia, PA, USA: Society for Industrial and Applied Mathematics, 2002.

[4] V. Volkov and J. Demmel, "LU, QR and Cholesky factorizations using vector capabilities of GPUs," Technical Report No. UCB/EECS, vol. 49, May 2008. [Online]. Available: http://parlab.eecs.berkeley.edu/publication/126

[5] S. Barrachina, M. Castillo, F. D. Igual, R. Mayo, E. S. Quintana-Ortí, and G. Quintana-Ortí, "Exploiting the capabilities of modern GPUs for dense matrix computations," Concurr. Comput. : Pract. Exper., vol. 21, pp. 2457-2477, December 2009. [Online]. Available: http://portal.acm.org/citation.cfm?id=1656506.1656515

[6] P. Benner, P. Ezzatti, E. S. Quintana, and A. Remón, "Using hybrid CPU-GPU platforms to accelerate the computation of the matrix sign function," in Lecture Notes in Computer Science, 7th Int. Workshop on Algorithms, Models and Tools for Parallel Computing on Heterogeneous Networks - HeteroPar'09, vol. 6043. Springer Berlin / Heidelberg, 2010, pp. 132-139.

[7] G. Golub and C. Van Loan, Matrix Computations, 3rd ed. Baltimore: Johns Hopkins University Press, 1996.

[8] P. Bientinesi, B. Gunter, and R. A. van de Geijn, "Families of algorithms related to the inversion of a symmetric positive definite matrix," ACM Trans. Math. Softw., vol. 35, pp. 3:1-3:22, July 2008. [Online]. Available: http://doi.acm.org/10.1145/1377603.1377606
[9] J. A. Gunnels, F. G. Gustavson, G. M. Henry, and R. A. van de Geijn, "Flame: Formal linear algebra methods environment," ACM Transactions on Mathematical Software, vol. 27, no. 4, pp. 422-455, Dec. 2001. [Online]. Available: http://doi.acm.org/10.1145/504210.504213

[10] P. Bientinesi, J. A. Gunnels, M. E. Myers, E. S. QuintanaOrtí, and R. A. van de Geijn, "The science of deriving dense linear algebra algorithms," ACM Trans. Math. Softw., vol. 31, pp. 1-26, March 2005. [Online]. Available: http://doi.acm.org/10.1145/1055531.1055532

[11] Intel Corporation., http://www. intel.com/.

[12] E. Anderson, Z. Bai, C. Bischof, L. S. Blackford, J. Demmel, J. J. Dongarra, J. DuCroz, S. Hammarling, A. Greenbaum, A. McKenney, and D. Sorensen, LAPACK Users' guide (third ed.). Philadelphia, PA, USA: Society for Industrial and Applied Mathematics, 1999.

[13] Nvidia Corporation, http://www.nvidia.com/cuda/. 